\title{
Pre-School Pediatric Cholesteatoma: 46 Cases
}

\author{
Ichiro Fukumoto ${ }^{1 *}$, Hiroki Koizumi², Yusuke Takata ${ }^{3}$, Seiichi Kadowaki², Yusuke Kimura ${ }^{4}$ and Hiroshi Sunose ${ }^{2}$ \\ ${ }^{1}$ Department of Otorhinolaryngology, Head and Neck Surgery, Chiba University Graduate School of Medicine, Japan
}

${ }^{2}$ Department of Otorhinolaryngology, Tokyo Women's Medical University Medical Center East, Japan

${ }^{3}$ Department of Otorhinolaryngology, Juntendo University Graduate School of Medicine, Japan

${ }^{4}$ Department of Otorhinolaryngology, Head and Neck Surgery, Nihon University School of Medicine, Japan

Submission: May 03, 2021; Published: May 18, 2021

*Corresponding author: Ichiro Fukumoto, Department of Otorhinolaryngology/Head and Neck Surgery, Chiba University Graduate School of Medicine, Chiba, Japan

\section{Abstract}

Pediatric cholesteatoma is classified into congenital cholesteatoma and acquired cholesteatoma. As congenital cholesteatoma has few symptoms, the cases are usually discovered with hearing loss during the examination during enrollment in an elementary school. Acquired cholesteatoma is associated with inflammatory changes and eustachian tube dysfunction of the middle ear, and it is said that most cases are more severe than congenital cholesteatoma. We analyzed 46 cases (47 ears) of pediatric cholesteatoma, diagnosed before attending school and operated upon. We also examined the discovery mechanism, classification, progress level, operative methods of cholesteatoma, postoperative hearing tests, and recurrences. Approximately $85 \%$ of these cases were of congenital cholesteatoma; additionally, approximately $15 \%$ had acquired cholesteatoma. As a result, there were no significant differences between congenital cholesteatoma cases and acquired cholesteatoma cases in terms of the degree of progression, hearing ability prognosis, and rate of recurrence. Moreover, the rate of recurrence and hearing ability prognoses were better than those in previous reports.

In general, there are several cases in which the cholesteatoma extends to anatomical blind spots such as the tympanic sinus or facial recess in pediatric cholesteatoma. Several papers have discussed the availability of middle ear surgeries using endoscopy (i.e., trans canal endoscopic ear surgery [TEES]). However, we performed middle ear surgery using microscopy (i.e., microscopic ear surgery [MES]) in all cases, and we have invented a way to find operation fields using procedures such as drilling the anterior wall of the external auditory canals.

In conclusion, we believe that polite dissection of the cholesteatoma matrix under the microscope is an important factor in the low rate of recurrence of cholesteatoma..

Keywords: Congenital cholesteatoma; Acquired cholesteatoma; Pediatric cholesteatoma; MES; TEES

\section{Introduction}

Middle ear cholesteatoma is classified roughly into congenital cholesteatoma and acquired cholesteatoma. Additionally, the acquired cholesteatoma is classified into three types: pars flaccida, pars tensa, and secondary types. As all cases of cholesteatoma are progressive diseases and cause complications, not only otorrhea and deafness, but also vertigo, deafness, facial nerve palsy, sigmoid sinus thrombosis and intracranial complications such as meningitis and epidural/subdural abscess [1], early diagnoses and early operations are required.

Pediatric cholesteatoma is classified into congenital cholesteatoma and acquired cholesteatoma. It is said that the congenital cholesteatoma is relatively rare and accounts for approximately $12.9 \%$ in all middle ear cholesteatomas [2]. Due of the lack of symptoms, such as otorrhea in congenital cholesteatoma, the cases are usually discovered for hearing loss at examination at enrollment in an elementary school. Therefore, congenital cholesteatoma before attending school is often confirmed accidentally by a mesotympanic white lesion in the otolaryngology outpatient department. Acquired cholesteatoma is associated with inflammatory changes and eustachian tube dysfunction in the middle ear. Compared with congenital cholesteatoma, the grades of disease are often severe [3]. As most cases of pediatric cholesteatoma are often diagnosed by a medical 
examination at school attendance, there are few articles about the cases that are diagnosed before attending school. We examined cases that were diagnosed before attending school and were operated on at this time.

In addition, there are numerous reports that trans canal endoscopic ear surgery (TEES) is effective because congenital cholesteatoma matrices often invaginate into the tympanic sinus and facial nerve recess, which are anatomical blind spaces in pediatric cholesteatoma [4]. Despite many reports of TEES, we performed microscopic ear surgery (MES) in all middle ear surgery cases in our department. Therefore, the aim of this study was to investigate the results of our operation via microscopy, such as the rate of recurrence and postoperative hearing levels in pre-school pediatric cholesteatoma.

\section{Materials and Methods}

We examined 46 cases (47 ears) who were diagnosed with cholesteatoma before enrollment at school in the Department of Otolaryngology, Tokyo Women's Medical University East Medical Center from June 2010 to January 2019. In total, 28 cases were boys, 18 cases were girls, and the average age of the 46 cases was 3.6 years old. In these cases, we examined the consultation opportunity, classification, progress level, operative method of cholesteatoma, postoperative hearing test, and recurrences. We classified all cases based on the JOS staging system for middle ear cholesteatoma (2015) [5]. Cholesteatomas that develop congenitally in the middle ear cavity and is non-continuous with the tympanic membrane and external auditory canal were classified as congenital cholesteatoma. We also classified the acquired cholesteatoma into the flaccid pars flaccida type, pars tensa type, and secondary type. Cholesteatomas occurring due to secondary development of cornified epithelium in the tympanic membrane and behind the handle of the malleus from the perforated margin of a perforation in the pars tensa were also classified as secondary cholesteatoma. Moreover, we evaluated the extension of cholesteatoma using the JOS staging system for middle ear cholesteatoma (2015) [5]. The postoperative hearing test results showed ossicular reconstruction and passed more than one year, and audiometric test results were intended for 27 cases. We judged three patterns as successful cases as below:

a. within $15 \mathrm{~dB}$ of air bone gap,

b. above $15 \mathrm{~dB}$ of hearing improvement, and

c. within $30 \mathrm{~dB}$ of hearing level,

based on the Guideline for Reporting Hearing Results in Middle Ear and Mastoid Surgery(2010). In addition, we classified the cases of recurrence into reconstruction recurrence and persistence recurrence as the mechanism of recurrence.

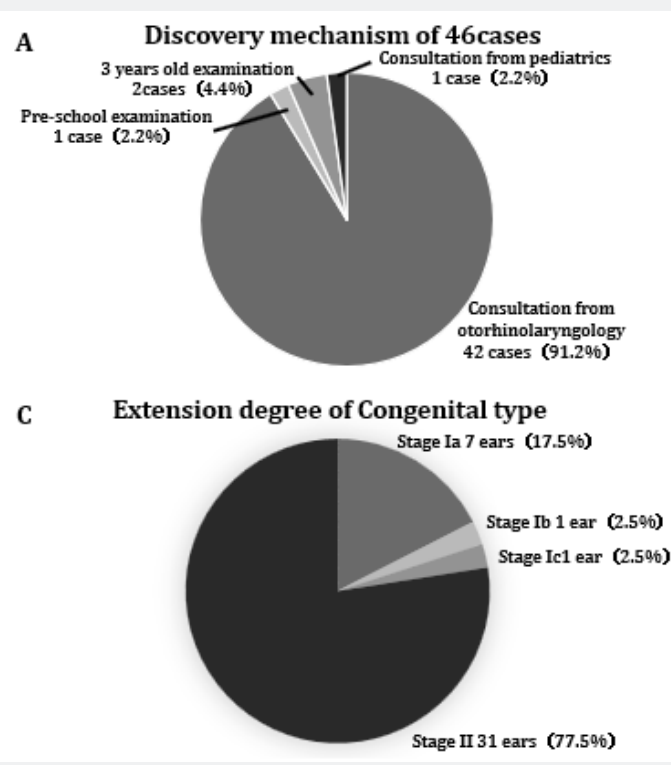

B

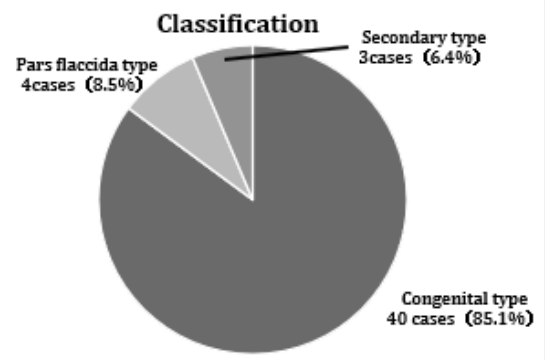

D

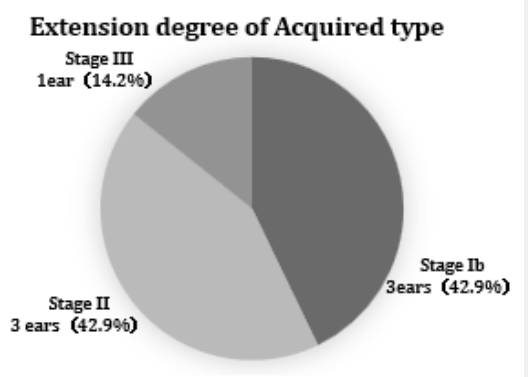

Figure 1: Forty-six cases (47 ears) diagnosed with middle ear cholesteatoma before attendance at school., A) Consultation opportunity: Forty-two out of 46 cases (91.2\%) were identified in the nearby otolaryngology outpatient department. One case was suspected to have hearing loss at a pre-school examination, and two cases were suspected of hearing loss in an examination at the age of 3 years. One case was introduced from the pediatric department. B) Classification: Seven ears of 40 ears (85.1\%) had congenital cholesteatoma. Seven ears had acquired cholesteatoma; in these cases, four cases were Pars flaccida type (8.5\%), and three cases were the secondary type(6.4\%). C) Staging of 40 patients with CC: Seven patients (17.5\%) had stage la (in the anterior tympanium), Stage $\mathrm{lb}$ (in the posterior tympanium), and Stage Ic (at both sites of the tympanium) were observed in one case (2.5\%). Additionally, 31 cases $(77.5 \%)$ were stage II, which extended to at least two lesions.D) Staging of 7 cases of acquired cholesteatoma: Three ears (42.9\%) were stage II, three ears were stage $\mathrm{lb}$, and only one case $(14.2 \%)$ had stage III lateral semicircular canal fistula. 
The opportunity to consult our department was obtained by consultation with other otorhinolaryngologists in more than $90 \%$ of cases. In one case involving a 3-year-old child, hearing loss was suspected at the examination; additionally, one patient who was referred from pediatrics was noted to have hearing loss during a pre-school medical examination (Figure 1A). In addition, 40 ears $(85.1 \%)$ showed congenital cholesteatoma, and the acquired cholesteatoma was seen in 7 cases (14.9\%). In acquired cholesteatoma, three cases were secondary cholesteatoma, and four cases were pars flaccida cholesteatoma (Figure 1B). For congenital cholesteatoma, 9 cases $(22.5 \%)$ were stage I, one lesion was extended, and 31 cases (77.5\%) were stage II, which extended to at least two lesions (Figure 1C). As for the acquired cholesteatoma, only one case was stage III with a lateral semicircular canal fistula; however, Stage I and Stage II were observed to occur in three cases (Figure 1D). There was no clear tendency denoting that the cases of acquired cholesteatoma had a more severe extension in our cases.

We performed single-stage operations in approximately $90 \%$ of cases (Figure 2A). In the cases in which cholesteatomas extended to the mastoid, only one patient underwent canal wall down (CWD) mastoidectomy (i.e., drilling of the posterior wall of the external auditory canal). Notably, other cases underwent canal wall up (CWU) mastoidectomy (i.e., preserving the posterior wall of the external auditory canal). In total, $12.8 \%$ of cases of extension of the cholesteatoma confined by the attic were treated with trans canal atticotomy (TCA). Cholesteatoma was confined to the tympanum in $42.6 \%$ of cases, and mastoidectomy was not performed (Figure 2B). Approximately $80 \%$ of the cases required sound conduction reconstruction, and type III reconstruction was required in 21 cases(44.6\%) and type IV in 17 cases(36.1\%) (Figure 2c).

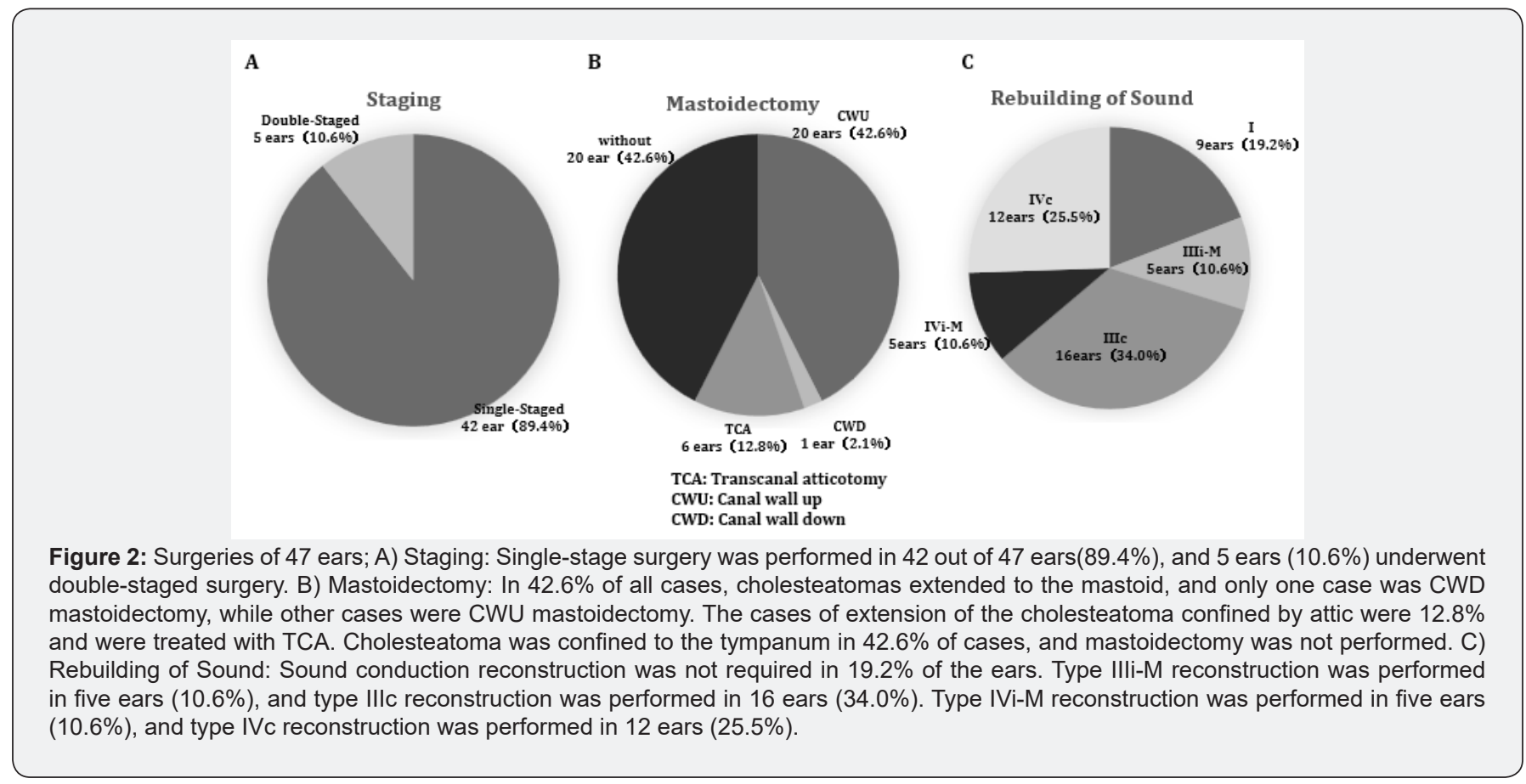

We evaluated hearing tests in 27 cases one year after sound conduction reconstruction; additionally, the success rate was $81.5 \%$ in 22 cases (Table 1A). The success rate was $81.8 \%(18 / 22$ cases) for congenital cholesteatoma and $80.0 \%$ (4/5 cases) of acquired cholesteatoma. However, the evaluation was difficult because the number of acquired cholesteatoma cases were few. There was no significant difference in the hearing prognoses between congenital cholesteatoma and acquired cholesteatoma (Table 1B).

Recurrences were found in six cases in 47 cases(12.8\%). Six cases were all advanced cases of stage II or stage III, and there were no recurrence cases in the case of Stage I (Table 2). The recurrences seemed to have several persistence-related recurrences in the previous report [6]; however, persistencerelated, and reconstitution-related recurrences occurred in three cases. Additionally, the recurrent style did not show any apparent difference (Table 2). Recurrences were observed in 5 ears out of $40(12.5 \%)$ of congenital cholesteatoma and in one case $(14.3 \%)$ of acquired cholesteatoma (Table 3). As there were few cases of acquired cholesteatoma, the evaluation was difficult; no major differences in the recurrence rate between congenital cholesteatoma and acquired cholesteatoma were found. 
Table 1: Hearing tests in 27 cases one year after sound conduction reconstruction.

\begin{tabular}{|c|c|}
\hline \multicolumn{1}{|c|}{ Table 1A } & Total 27 ears \\
\hline Hearing Test after one year from operation & 9 ears \\
\hline Within 15dB Air-Bone gap & 6 ears \\
\hline Above 15dB of improvement from pre-operation & 22 ears ears(81.5\%) \\
\hline Within 30dB of hearing test & 5 ears(18.5\%) \\
\hline Correspond to either (Success) & Total 27 ears \\
\hline Correspond to non (Failure) & $18 / 22$ ears(81.8\%) \\
\hline Successful rate & $4 / 5$ ears(80.0\%) \\
\hline congenital cholesteatoma & \\
\hline acquired cholesteatoma & \\
\hline
\end{tabular}

Table 2: Cases of Recurrence.

\begin{tabular}{|c|c|c|c|c|c|c|c|}
\hline Case & Age & Sex & Classification & Extension & Staging & Recurrent Style & Recurrent Part \\
\hline 1 & 3 & F & Congenital & Stage II & Single & Persistence & Mesotympanic \\
\hline 2 & 6 & F & Congenital & Stage II & Double & Persistence & Mesotympanic \\
\hline 3 & 5 & F & Acquired & Stage III & Single & Persistence & attic and mastoid \\
\hline 4 & 2 & F & Congenital & Stage II & Double & Reconstitution & attic \\
\hline 5 & 4 & F & Congenital & Stage II & Single & Reconstitution & attic and mastoid \\
\hline 6 & 6 & M & Congenital & Stage II & Single & Reconstitution & attic \\
\hline
\end{tabular}

Table 3: Recurrent rate.

\begin{tabular}{|c|c|}
\hline congenital & $5 / 40 \operatorname{ears}(12.5 \%)$ \\
\hline acquired & $1 / 7$ ears(14.3\%) \\
\hline
\end{tabular}

\section{Discussion}

There have been several discussions about the surgical method for middle ear surgery. There are numerous papers comparing the methods of mastoidectomy to determine the superior method: CWD or CWU [7]. In our department, we often choose CWD mastoidectomy based on the rate of recurrences in most adult cases, except in cases where pneumatization of the mastoid is highly developed. Past reports concluded that CWD mastoidectomy can obtain a low recurrence rate and a high rate of trouble-free ear in the long term in pediatric cholesteatoma [8]. However, in pediatric cases, we choose CWU mastoidectomy because of the undeveloped pediatric temporal bone; this method helps to maintain the form of the external auditory canal and the patients can enjoy swimming. In this study, we performed CWU mastoidectomy, except in one case. One case involved severe cases of stage III with lateral semicircular canal fistula, which recurred several times. About the number of operations, there are many advantages to performing a double-staged operation.

Silicon sheets can be inserted to prevent tympanic adhesion during the first surgery and confirm the presence of recurrence at the second stage of surgery. Nevertheless, we performed singlestaged operations in $89.4 \%$ of cases, and the rate of recurrence was only $12.8 \%$ in pediatric cholesteatoma cases. This rate was better than that in previous reports [6]. In general, the postoperative prognosis of hearing ability was good in pediatric cholesteatoma, and we were able to obtain a success rate of $81.5 \%$. We performed all the middle ear operations in our department via microscopy using the MES approach. However, in the recent years, the number of operations performed using endoscopy (i.e., TEES) has increased. There are many reports of TEES $[9,10]$, and the effectiveness of MES and TEES has been discussed [11].

In congenital cholesteatoma cases, the matrix of the cholesteatoma may extend to the anatomical blind spaces, including the tympanic sinus or facial recess. In these cases, TEES may be effective [12]. Moreover, TEES should remain in effect in a narrow external auditory canal, such as pediatric cases [13]. The field of TEES is good; however, the surgical procedure is not at all brief, and adverse events increase in inexperienced operators [14]. We use microscopy techniques to open the external auditory canal, such as drilling the anterior wall and finding a good field of anatomical blind spaces. We used an endoscope to confirm whether the anatomical blind spot had persistence of the cholesteatoma matrix. We concluded that a polite dissection of the cholesteatoma matrix under microscopy was the key factor in the low recurrence rate. 


\section{Conclusion}

We examined 46 cases (47 ears) with cholesteatomas who were diagnosed and operated upon before attending elementary school. In most cases, we could preserve the posterior walls of the external auditory canals and perform single-staged operations. In all cases, we performed the surgeries using microscopy, and we had no cases that required endoscopy. However, we were able to obtain a low rate of recurrence and a good hearing prognosis.

\section{Acknowledgement}

This study was supported by the KAKENHI, 18K16866.

\section{References}

1. Kennedy KL, Singh AK (2020) Middle Ear Cholesteatoma, StatPearls, Treasure Island, Stat Pearls Publishing.

2. Morita Y, Tono T, Sakagami M, Yamamoto Y, Matsuda K, et al. (2019) Nationwide survey of congenital cholesteatoma using staging and classification criteria for middle ear cholesteatoma proposed by the Japan Otological Society. Auris Nasus Larynx 46(3): 346-352.

3. Morita Y, Yamamoto Y, Oshima S, Takahashi K, Takahashi S (2016) Pediatric middle ear cholesteatoma: the comparative study of congenital cholesteatoma and acquired cholesteatoma. Eur Arch Otorhinolaryngol 273(5): 1155-1160.

4. Park JH, Ahn J, Moon IJ (2018) Transcanal Endoscopic Ear Surgery for Congenital Cholesteatoma. Clin Exp Otorhinolaryngol 11(4): 233-241.

5. Tono T, Sakagami M, Kojima H, Yamamoto Y, Matsuda K, et al. (2017) Staging and classification criteria for middle ear cholesteatoma proposed by the Japan Otological Society. Auris Nasus Larynx 44(2):
$135-140$.

6. Morita Y, Takahashi K, Izumi S, Kubota Y, Ohshima S, et al. (2017) Risk Factors of Recurrence in Pediatric Congenital Cholesteatoma. Otol Neurotol 38(10): 1463-1469.

7. Karamert R, Eravcı FC, Cebeci S, Düzlü M, Zorlu ME, et al. (2019) Canal wall down versus canal wall up surgeries in the treatment of middle ear cholesteatoma. Turk J Med Sci 49(5): 1426-1432.

8. Trinidade A, Skingsley A, Yung MW (2015) Pediatric cholesteatoma surgery using a single-staged canal wall down approach: results of a 5-year longitudinal study. Otol Neurotol 36(1): 82-85.

9. Imai T, Nishiike $\mathrm{S}$, Oshima $\mathrm{K}$, Tanaka $\mathrm{H}$, Tsuruta $\mathrm{Y}$, et al. (2017) The resected area of the posterior wall of the external auditory canal during transcanal endoscopic ear surgery for cholesteatoma. Auris Nasus Larynx 44(2): 141-146.

10. Kapadiya M, Tarabichi M (2019) An overview of endoscopic ear surgery in 2018. Laryngoscope Investig Otolaryngol 4(3): 365-373.

11. Hsu YC, Kuo CL, Huang TC (2018) A retrospective comparative study of endoscopic and microscopic Tympanoplasty. J Otolaryngol Head Neck Surg 47(1): 44.

12. Hunter JB, Zuniga MG, Sweeney AD, Bertrand NM, Wanna GB, et al (2016) Pediatric Endoscopic Cholesteatoma Surgery. Otolaryngol Head Neck Surg 154(6): 1121-1127.

13. Ito T, Kubota T, Watanabe T, Futai K, Furukawa T, et al. (2015) Transcanal endoscopic ear surgery for pediatric population with a narrow external auditory canal. Int J Pediatr Otorhinolaryngol 79(12): 2265-2269.

14. Dedmon MM, Kozin ED, Lee DJ (2015) Development of a Temporal Bone Model for Transcanal Endoscopic Ear Surgery. Otolaryngol Head Neck Surg 153(4): 613-615.

\section{Your next submission with Juniper Publishers will reach you the below assets}

- Quality Editorial service

- Swift Peer Review

- Reprints availability

- E-prints Service

- Manuscript Podcast for convenient understanding

- Global attainment for your research

- Manuscript accessibility in different formats

( Pdf, E-pub, Full Text, Audio)

- Unceasing customer service

Track the below URL for one-step submission https://juniperpublishers.com/online-submission.php 